
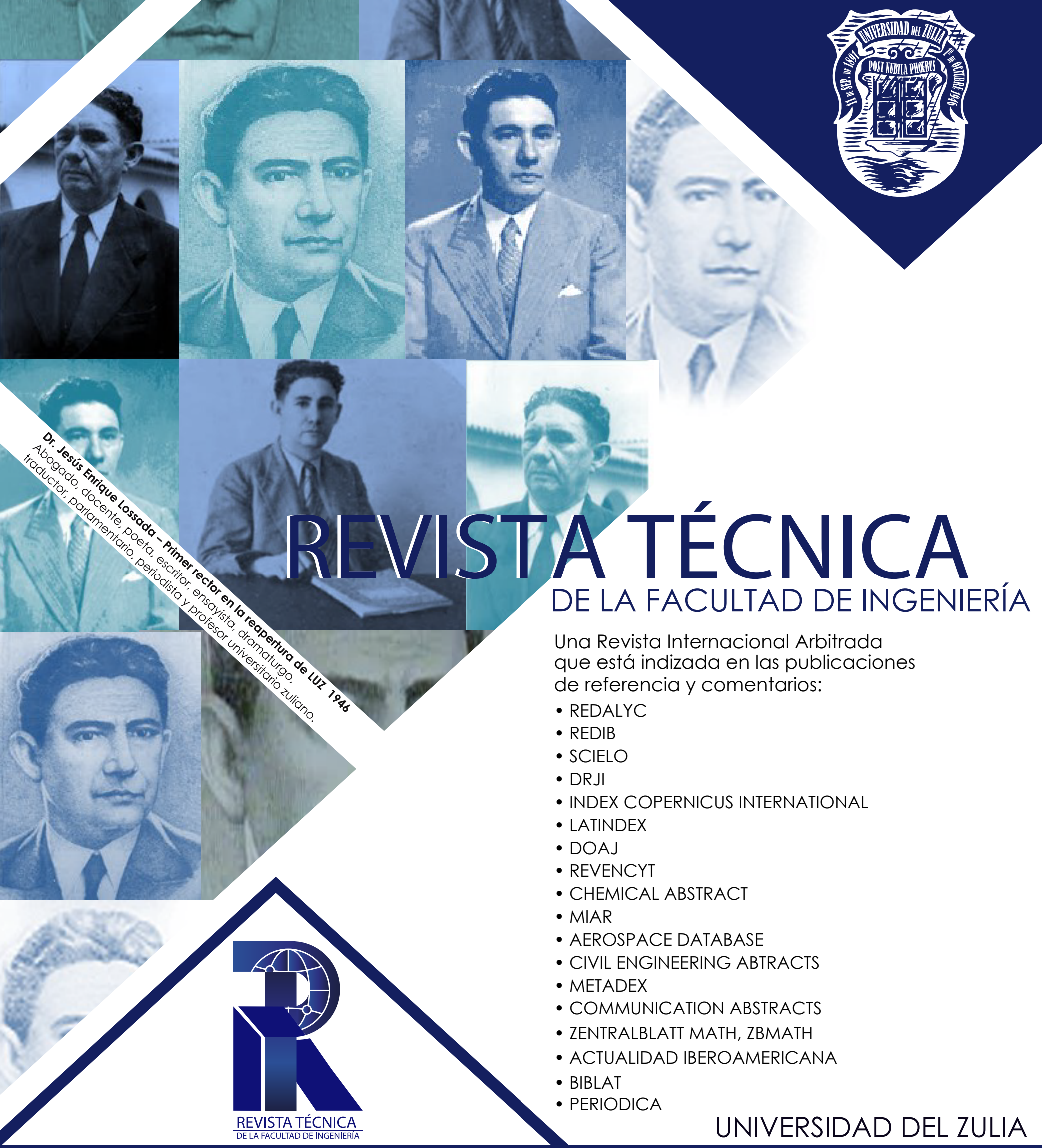

Una Revista Internacional Arbitrada que está indizada en las publicaciones de referencia y comentarios:

- REDALYC

- REDIB

- SCIELO

- DRJI

- INDEX COPERNICUS INTERNATIONAL

- LATINDEX

- DOAJ

- REVENCYT

- CHEMICAL ABSTRACT

- MIAR

- AEROSPACE DATABASE

- CIVIL ENGINEERING ABTRACTS

- METADEX

- COMMUNICATION ABSTRACTS

- ZENTRALBLATT MATH, ZBMATH

- ACTUALIDAD IBEROAMERICANA

- BIBLAT

- PERIODICA 


\title{
Análisis de la Resistencia a Corte de Conectores Tipo Ángulo en Losas de Hormigón Armado sobre Estructura Metálica
}

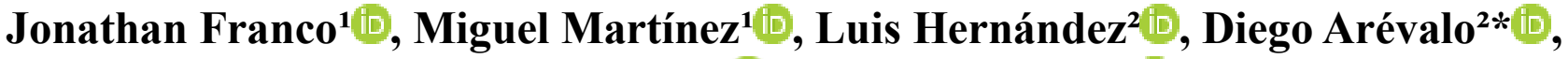 \\ Melisa Herrera' ${ }^{2}$, Christian Gómez ${ }^{2}$ (D) \\ ${ }^{1}$ Departamento de Ingeniería Civil, Facultad de Ingeniería Civil y Ambiental, Escuela Politécnica \\ Nacional, Quito, Ecuador. \\ ${ }^{2}$ Centro de Investigación de la Vivienda (CIV-EPN), Facultad de Ingeniería Civil y Ambiental, \\ Escuela Politécnica Nacional, Quito, Ecuador. \\ *Autor de correspondencia: diego.arevalo@epn.edu.ec
}

https://doi.org/10.22209/rt.v45n1a04

Recepción: 24 de febrero 2021 | Aceptación: 11 de octubre de 2021 | Publicación: 29 de diciembre de 2021

\section{Resumen}

Durante años se ha venido construyendo losas de hormigón sobre vigas de acero, sin embargo, el no considerar la trasmisión de esfuerzos de corte entre ellas, ocasiona que los elementos trabajen por separado. Para asegurar la transmisión de esfuerzos, la carga debe transmitirse mediante conectores mecánicos. En la construcción comúnmente se utilizan pernos stud, perfiles angulares y tornillos como conectores, aunque no todos se encuentren normados. El presente trabajo analiza el comportamiento de conectores de corte tipo ángulo, cuando están localizados de diferente manera a la expuesta en códigos de diseño. Para ello, se consideraron conectores de corte tipo ángulo, colocados de manera perpendicular respecto a la cara del patín de la viga de acero, analizando dos tipos de posiciones (45 y $90^{\circ}$ referente al eje longitudinal del patín). Para comprobar cuál tiene un comportamiento y resistencia adecuada, se realizaron ensayos push-out, con un total de 6 probetas por posición. Las curvas fuerza vs. deslizamiento obtenidas del conector a $45^{\circ}$, presentaron un comportamiento más homogéneo en todos los parámetros analizados, en comparación con las curvas obtenidas para el conector a $90^{\circ}$. Su óptimo rendimiento hace que el conector a $45^{\circ}$ sea el más adecuado para implementación.

Palabras clave: resistencia a corte; conectores de corte; conector tipo ángulo; ensayo push-out.

\section{Shear Strength Analysis of Angle-type connectors in Reinforced Concrete Slabs on a Metallic Structure}

\begin{abstract}
For years concrete slabs have been built on steel beams, however, not considering the transmission of shear forces between them, causes the elements to work separately. To ensure the transmission of forces, the load must be transmitted through mechanical connectors. In construction, stud bolts, angle profiles and screws are commonly used as connectors, although not all of them are standardized. The present work analyzes the behavior of angle shear connectors when they are located in a different way than stated in design codes. For this study, angle share connectors were considered placed perpendicular to the face of the steel beam skid; analyzing two types of positions (45 and $90^{\circ}$ referring to the longitudinal axis of the flange). In order to check which one has adequate behavior and resistance, push-out tests were carried out, with a total of 6 specimens per position. The shear force vs. slip curves obtained from the connector at $45^{\circ}$ showed a more homogeneous behavior in all the parameters analyzed compared to the curves obtained for the connector at $90^{\circ}$. Its optimal performance makes the $45^{\circ}$ connector the most suitable for implementation.
\end{abstract}

Key words: shear resistance; shear connector; angle connector; push-out test. 


\title{
Análise da Resistência ao Cisalhamento de Conectores do Tipo Angular em Lajes de Concreto Armado em Estrutura Metálica
}

\begin{abstract}
Resumo
Durante anos, lajes de concreto foram construídas por cima das vigas de aço, porém, a desconsideração da transmissão de forças de cisalhamento entre elas, faz com que os elementos funcionanm separadamente. Para garantir a transmissão dos esforços, a carga deve ser transmitida por meio de conectores mecânicos. Pinos, barras angulares e parafusos são normalmente usados em construção como conectores, embora nem todos sejam padronizados. O presente trabalho analisa o comportamento de conectores de cisalhamento do tipo ângulo, quando estão localizados de forma diferente daquela declarada nos códigos de projeto. Para isso, foram seleccionados conectores de corte do tipo angulares, colocados perpendicularmente à face do patim da viga de aço, analisando dois tipos de posições (45 e $90^{\circ}$ em relação ao eixo longitudinal do patim). Para verificar, se apresenta o comportamento e a resistência adequados, para os testes de push-out, foram utilizados um total de 6 corpos de prova por posição. A força vs. o escorregamento obtido do conector a $45^{\circ}$, apresentou um comportamento mais homogêneo em todos os parâmetros analisados, em comparação com como curvas obtidas para o conector a $90^{\circ}$. Seu desempenho ideal torna o conector de $45^{\circ}$ o mais adequado para implantação.
\end{abstract}

Palavras-chave: resistência ao corte; conectores de corte; conector tipo ângulo; teste de push-out.

\section{Introducción}

Los conectores de corte que unen una viga compuesta (losa de hormigón y viga metálica) ayudan a transmitir de mejor manera los esfuerzos cortantes, aumentando la capacidad a corte entre 30 y $50 \%$. Para que esto suceda los conectores deben estar soldados al patín de la viga (McCormac y Csernak, 2013; Deng et al., 2019). Entre los tipos de conectores, están: pernos stud, varillas corrugadas, espirales, angulares y tornillos (Crisafulli, 2018). El estudio experimental de los conectores de corte se puede realizar mediante varios métodos, pero el más utilizado en el área de investigación es el ensayo push-out. Este ensayo consiste en la aplicación de carga y descarga, durante 25 ciclos para después llevar la probeta a la carga de falla. Este tipo de ensayo genera resultados favorables para el análisis de los conectores de corte (UNE EN 1994 1-1, 2013).

El American Institute of Steel Construction (AISC) determina los criterios de diseño del conector tipo stud, considerando la resistencia (ANSI/AISC 360-16, 2016). Sin embargo, esta resistencia puede ser afectada por varios factores, como son: la corrosión por el paso de los años (Chen et al., 2016), el refuerzo en la losa y la geometría de los conectores que no están dentro de los criterios de diseños establecidos por el AISC (Horita et al., 2012). Deng et al. (2019) ensayaron 3 tipos de conectores en losas de hormigón maciza, siendo uno de estos un conector tipo ángulo, analizaron la ductilidad, la resistencia a corte, la rigidez y su degradación para determinar el comportamiento de cada uno de los conectores, así pudieron determinar que el conector tipo ángulo fue el segundo con un comportamiento más adecuado. Shariati et al. $(2014 ; 2016)$ estudiaron conectores de corte tipo ángulo en losas de hormigón maciza, con los resultados obtenidos del ensayo push-out, determinaron una ecuación para su diseño, donde obtuvieron resultados favorables con la ecuación propuesta.

El presente trabajo analiza el comportamiento de conectores de corte tipo ángulo, cuando están localizados de diferente manera a la expuesta en códigos de diseño. Para ello, se consideraron conectores de corte tipo ángulo, colocados de manera perpendicular respecto a la cara del patín de la viga de acero, analizando dos tipos de posiciones (45 y $90^{\circ}$ referente al eje longitudinal del patín), como se muestra en la Figura 1, mediante el ensayo de probetas push-out no estandarizadas y siguiendo el protocolo de carga dispuesto por el Eurocódigo 4 (UNE EN 1994 1-1, 2013). Para ello, se analizaron las propiedades del sistema del conector, tales como: ductilidad, tenacidad y rigidez. También se determinó la posición del conector que presentaba un mejor comportamiento y se realizó una comparación de la resistencia del conector con respecto a fórmulas planteadas por los códigos internacionales e investigaciones previas. 


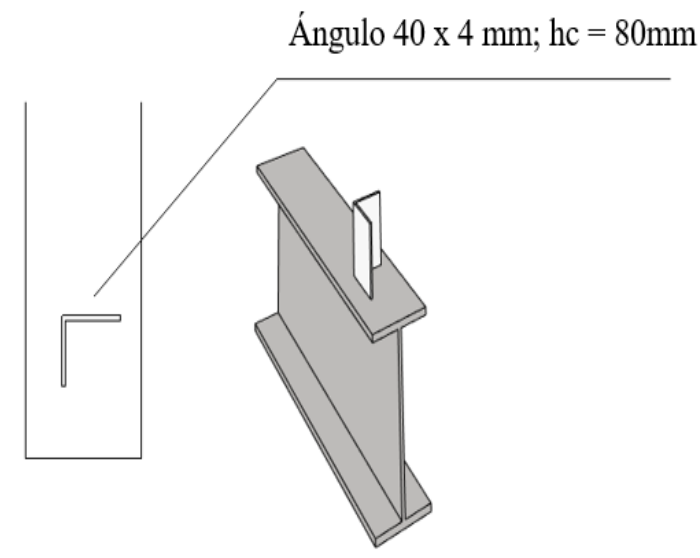

a) Conector $90^{\circ}$

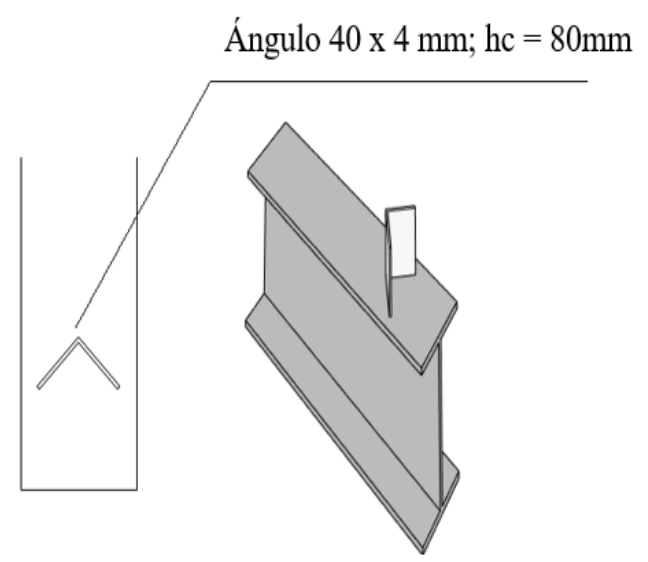

b) Conector $45^{\circ}$

Figura 1. Detalle de conectores tipo ángulo colocados de manera perpendicular respecto a la cara del patín de la viga de acero, en posición a 90 y $45^{\circ}$.

\section{Materiales y Métodos}

\section{Ensayo push-out y probetas de ensayo}

El ensayo push-out es uno de los más empleados para el estudio de la resistencia de los conectores, donde se obtienen curvas fuerza versus deslizamiento para cada conector. El Eurocódigo 4 (UNE-EN 1994 1-1, 2013) normaliza este tipo de ensayo con su probeta estandarizada, para pernos stud con un mínimo de 3 probetas para ensayar, sin embargo, se pueden realizar los ensayos con probetas no estandarizada. Por tal razón, en este trabajo se emplearon 6 probetas no estandarizadas para realizar las pruebas, donde, el conector propuesto para el análisis fue un ángulo de dimensiones de $40 \times 4 \mathrm{~mm}$, ubicado a 45 y $90^{\circ}$, como muestra la Figura 1, con una altura de $80 \mathrm{~mm}$. La geometría de la losa estuvo basada en investigaciones previas (Molina y Hurtado, 2011; Pavlović et al., 2013; Shariati et al., 2014), con dimensiones de 300 x 400 x $110 \mathrm{~mm}$. Se empleó una viga tipo I con una geometría de 200 x 100 × 6 × $8 \mathrm{~mm}$, que une las dos losas para conformar la probeta push-out, la cual contó con una longitud de 400 $\mathrm{mm}$, dimensión similar a la longitud de las losas de hormigón. La configuración de las probetas push-out se muestra en la Figura 2, así como sus dimensiones.

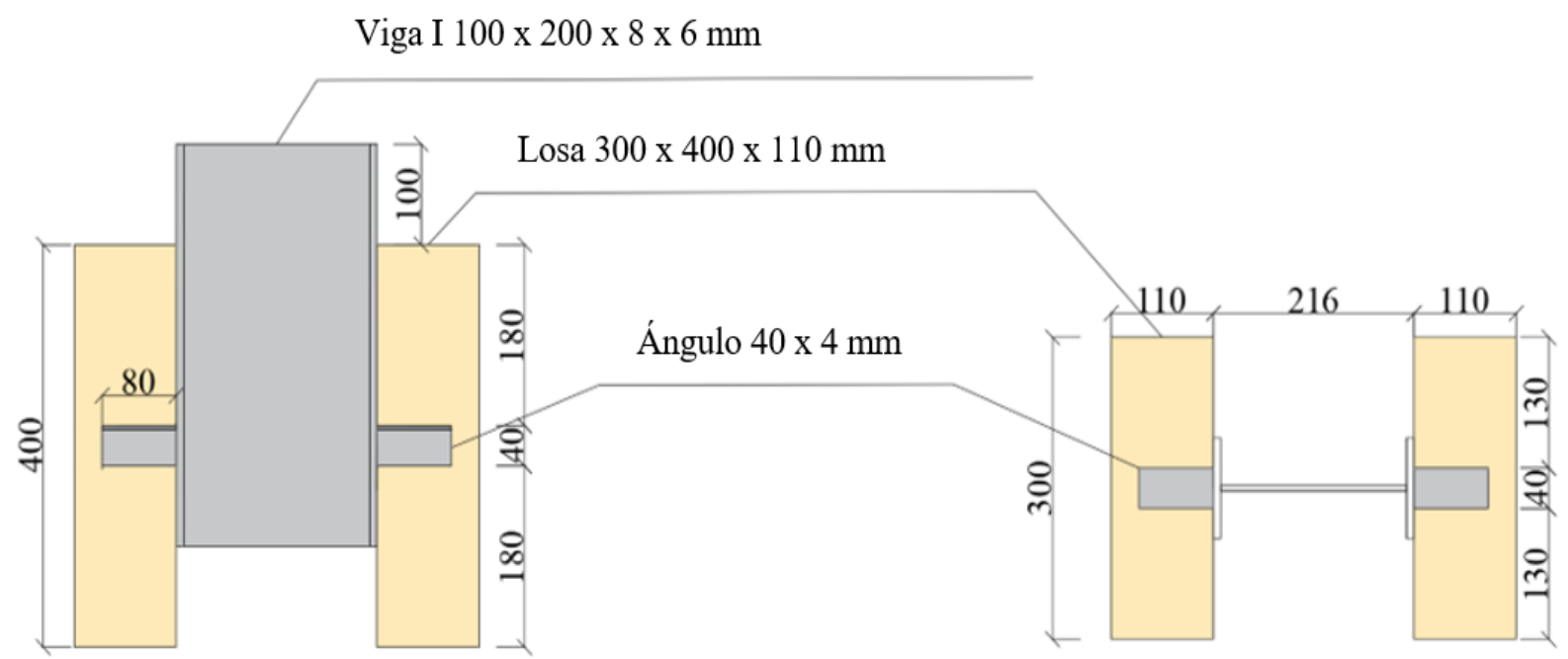

Vista en elevación

\section{Vista en planta}

Figura 2. Detalle de probetas utilizadas en ensayo push-out. 


\section{Protocolo de ensayo}

El protocolo de ensayo se dividió en dos etapas: la primera, denominada pre-carga, y la segunda, carga formal (Deng et al., 2019), como se muestra en la Figura 3. La precarga consistió en la aplicación de carga y descarga durante 25 ciclos entre un rango de 5 a $40 \%$ de la capacidad máxima a corte de los conectores. La carga formal partió en el ciclo 26, cuando la descarga alcanzaba el $5 \%$ de su valor, y se llevó la probeta push-out a la falla de rotura en un lapso no menor a $15 \mathrm{~min}$, o hasta que la curva había descendido en un $20 \%$ de la capacidad máxima a corte de los conectores (UNE EN 1994 1-1, 2013).

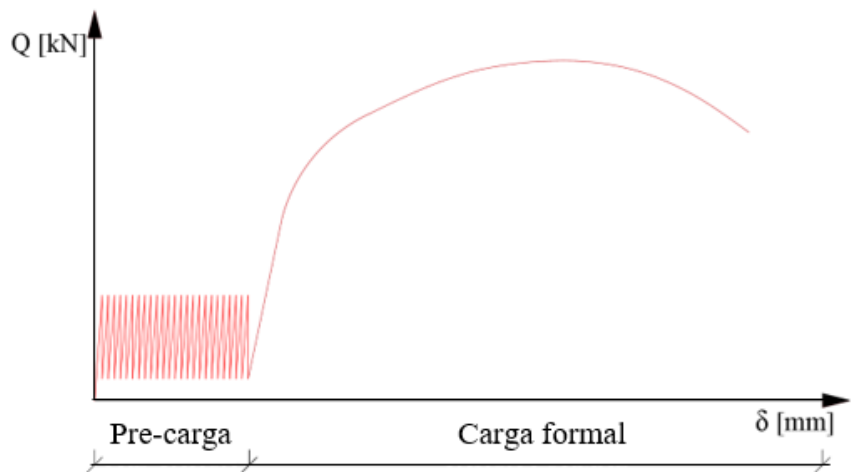

Figura 3. Etapas del protocolo de ensayo push-out (Deng et al., 2019).

\section{Instrumentación}

Existen dos parámetros fundamentales que se deben medir mientras se realiza el ensayo push-out, estos son: la carga aplicada a la probeta push-out y el deslizamiento que existe entre la losa y la viga (UNE EN 1994 1-1, 2013). Para medir la carga se empleó un gato hidráulico con capacidad de $980 \mathrm{kN}$. Se midieron los desplazamientos mediante los transductores de desplazamiento lineal variable (LVDT), como se muestra en la Figura 4.

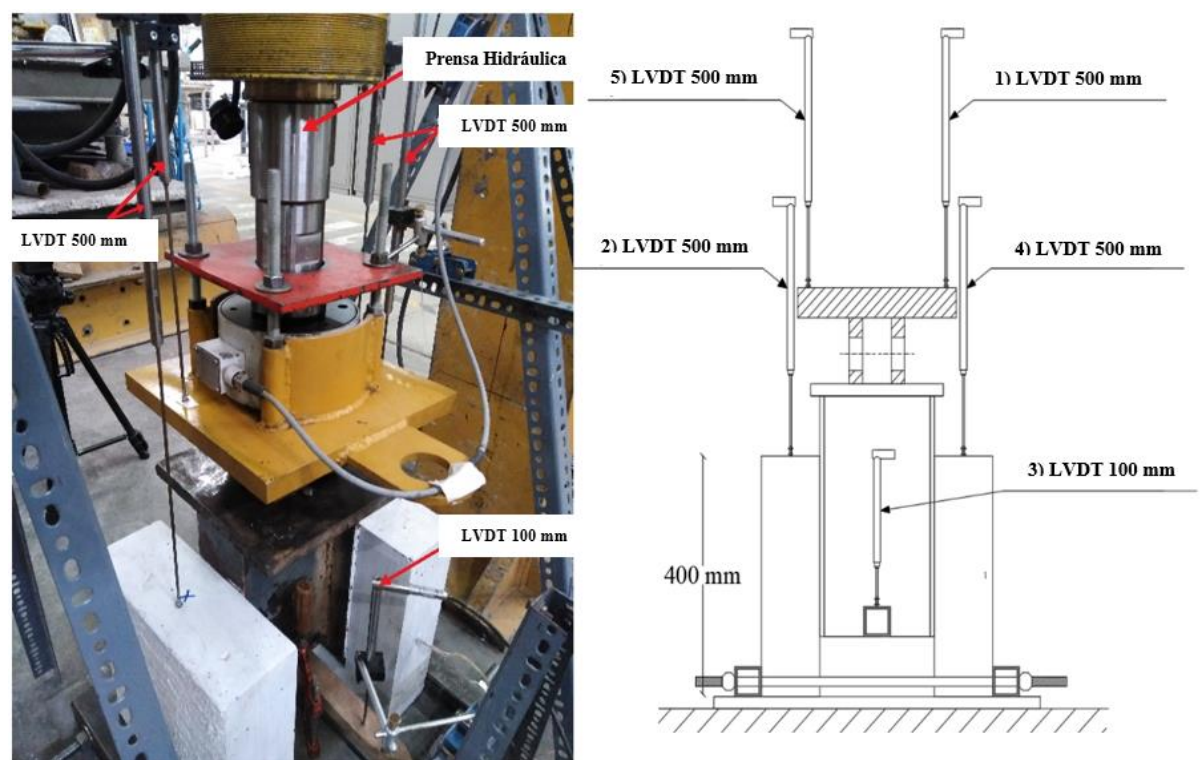

Figura 4. Instrumentación de probetas para ensayo push-out. LVDT: transductor de desplazamiento lineal variable.

\section{Propiedades de los materiales}

La resistencia se obtuvo del ensayo a compresión de cilindros de hormigón a los 28 días de edad, la cual fue de 26,03 MPa, y se obtuvo un módulo de elasticidad de 21,16 GPa a los 55 días de edad, realizando el mismo tipo de ensayo. Se realizaron ensayos a tracción con placas extraídas del ángulo de 40 x 4 mm, los cuales exhibieron un esfuerzo a tracción de $339 \mathrm{MPa}$. 


\section{Resultados y Discusión}

\section{Curva carga-deslizamiento}

Para analizar las propiedades mecánicas del conector tipo ángulo, se utilizó la curva carga-deslizamiento dispuesta en el Eurocódigo 4 (UNE EN 1994 1-1, 2013); por ende, la curva contó con los datos de la parte de la carga formal (Deng et al., 2019; Hällmark et al., 2019). Como se observa en la Figura 5, uno de estos datos eran las cargas obtenidas del ensayo, las cuales se analizaron por conector (Molina y Hurtado, 2011; Bamaga et al., 2019). Además, se tomó en cuenta que el deslizamiento obtenido es entre la losa y la viga, de acuerdo con el Eurocódigo 4 (UNE EN 1994 1-1, 2013). En la Figura 5 se muestra la carga máxima $\left(Q_{m a ́ x}\right)$, la resistencia carácteristica a corte $\left(P_{R K}\right)$ y el deslizamiento último $\left(\delta_{u}\right)$.

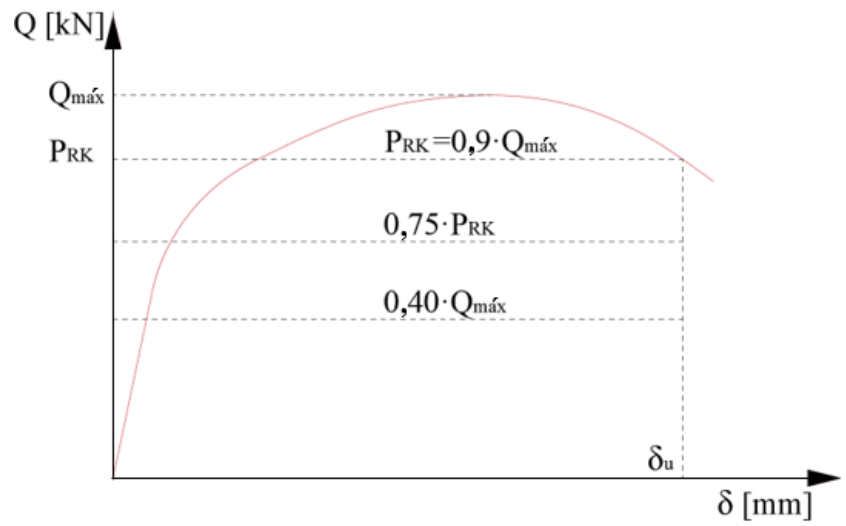

Figura 5. Valores de carga que se pueden obtener de la curva carga-desplazamiento.

Los conectores a $45^{\circ}$ presentaron un comportamiento lineal para la parte inicial de la curva, como se lo puede apreciar (Figura 5). La zona plástica de las curvas mostró un cambio de pendiente continuo y no abrupto, como se destaca en la Figura 6. También se observó que sus curvas prácticamente están juntas, lo cual indica que el comportamiento de estos conectores a $45^{\circ}$ son homogéneos.

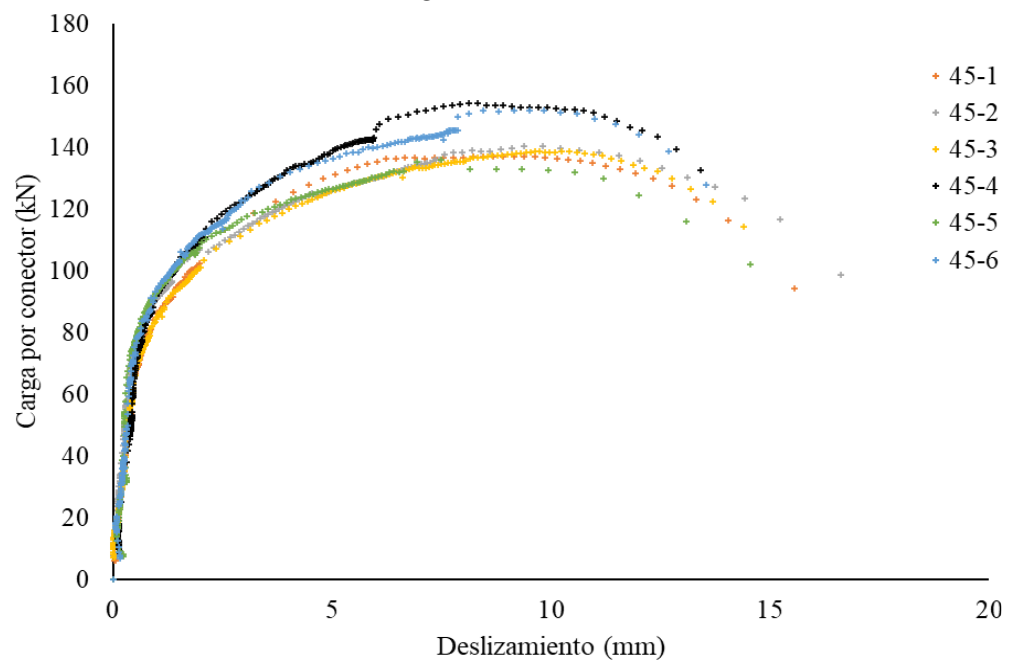

Figura 6. Curva carga-deslizamiento obtenida de ensayos push-out en conectores de corte tipo ángulo a $45^{\circ}$.

Las curvas para los conectores a $90^{\circ}$ se muestran en la Figura 7. Estos resultados presentaron curvas con poco decaimiento en la parte final de la zona plástica, debido a la repentina falla entre la unión del conector con la viga, pese a que la zona elástica tuvo una tendencia lineal. El ensayo 90-1 presentó un comportamiento atípico con relación a los demás ensayos, con una curva diferente una carga máxima mayor.

Los valores que se pueden obtener de las curvas presentadas en las Figuras 6 y 7, se incluyen en la Tabla 1. $Q_{m a ́ x}$ con su valor medio $\overline{Q_{m a ́ x}}, P_{R K}$ y su valor medio $\overline{P_{R K}}$, y $\delta_{u}$ con su respectivo valor medio $\overline{\delta_{u}}$. Los conectores 
exhibieron cargas máximas medias similares.

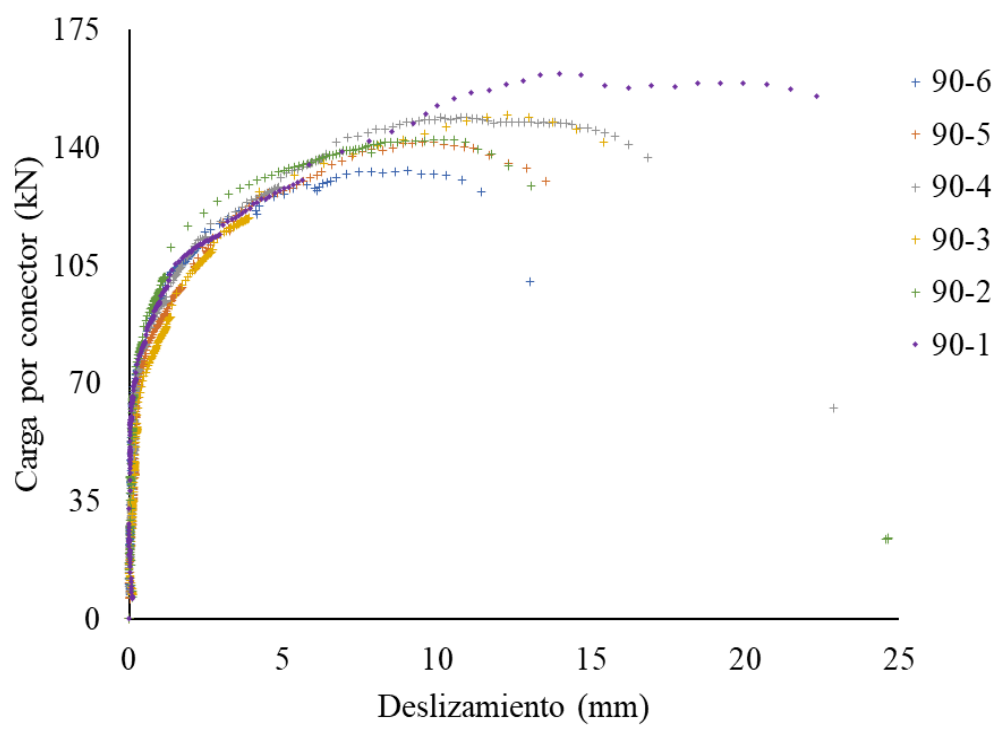

Figura 7. Curva carga-deslizamiento obtenida de ensayos push-out en conectores de corte tipo ángulo en posición a $90^{\circ}$.

Tabla 1. Resultados del ensayo push-out en conectores de corte tipo ángulo.

\begin{tabular}{|c|c|c|c|c|c|c|}
\hline $\mathbf{N}^{\mathbf{o}}$ & $\begin{array}{l}Q_{\text {máx }} \\
(\mathbf{k N})\end{array}$ & $\begin{array}{l}\overline{Q_{m a ́ x}} \\
(\mathrm{kN})\end{array}$ & $\begin{array}{c}P_{R K} \\
(\mathbf{k N})\end{array}$ & $\begin{array}{l}\overline{P_{R K}} \\
(\mathbf{k N})\end{array}$ & $\begin{array}{c}\delta_{u} \\
(\mathbf{m m})\end{array}$ & $\begin{array}{c}\overline{\delta_{u}} \\
(\mathbf{m m})\end{array}$ \\
\hline \multicolumn{7}{|c|}{ Conector a $45^{\circ}$} \\
\hline $45-1$ & 137,18 & \multirow{6}{*}{143,20} & 123,47 & \multirow{6}{*}{128,88} & 13,27 & \multirow{6}{*}{13,11} \\
\hline $45-2$ & 140,54 & & 126,49 & & 14,04 & \\
\hline $45-3$ & 138,86 & & 124,97 & & 13,37 & \\
\hline $45-4$ & 154,41 & & 138,97 & & 12,89 & \\
\hline $45-5$ & 136,02 & & 122,41 & & 12,28 & \\
\hline $45-6$ & 152,17 & & 136,95 & & 12,81 & \\
\hline \multicolumn{7}{|c|}{ Conector a $90^{\circ}$} \\
\hline $90-1$ & 161,76 & \multirow{6}{*}{146,13} & 155,01 & & 25,62 & \multirow{6}{*}{16,5} \\
\hline $90-2$ & 142,16 & & 128,37 & & 13,10 & \\
\hline $90-3$ & 149,64 & & 141,64 & 12641 & 17,03 & \\
\hline $90-4$ & 148,77 & & 136,87 & 150,42 & 17,08 & \\
\hline $90-5$ & 141,57 & & 130,03 & & 14,00 & \\
\hline $90-6$ & 132,90 & & 126,62 & & 11,86 & \\
\hline
\end{tabular}

Qmáx: carga máxima, $\overline{Q_{\text {máx }}}$ : valor medio de Qmáx, $\mathrm{P}_{\mathrm{RK}}$ : resistencia carácteristica a corte, $\delta_{u}$ : deslizamiento último.

\section{Cargas máximas y resistencia característica}

Para obtener $P_{R K}$ de los 6 ensayos, se analizaron las $Q_{m a ́ x}$ por cada conector. Este análisis lo estipula el Eurocódigo 4 (UNE EN 1994 1-1, 2013) e indica que cada una de las cargas máximas por conector no debe exceder en un $\pm 10 \%$ de la media de los 6 ensayos realizados. Si no se cumplen con este límite, las cargas máximas deben ser reducidas mediante modelos estadísticos, establecidos por el anexo D del Eurocódigo 0 (UNE EN 1990 1-1, 2019). De las dos posiciones analizadas, el conector a $90^{\circ}$ presentó una probeta con un comportamiento atípico, el cual no cumple con lo establecido por el Eurocódigo 4 (UNE EN 1994 1-1, 2013). Por tal motivo, se empleó el modelo estadístico para el cálculo de la resistencia, recomendado en el anexo D, método A, expresión D.17 del Eurocódigo 0 (UNE EN 1990 1-1, 2019). Una vez aplicado este método, se obtuvo un factor de reducción, $\mathrm{n}_{\mathrm{k}}$, de 0,848 para las cargas del conector a $90^{\circ}$. Este factor se aplicó para la carga del conector 90-1, quedando un valor de 137,17 kN. En la Tabla 2 se muestra el porcentaje resultante entre la carga media, con respecto a cada una de las cargas de los conectores. Estos valores cumplen con el límite, por ende, se puede determinar el valor del $P_{R K}$. 
Tabla 2. Cargas máximas corregidas con modelo estadístico en conectores tipo ángulo a $90^{\circ}$.

\begin{tabular}{lcc}
\hline $\boldsymbol{Q}_{\text {máx }}(\mathbf{k N )}$ & $\begin{array}{c}\overline{\boldsymbol{Q}_{\text {máx }}} \\
(\mathbf{k N})\end{array}$ & Error (\%) \\
\hline 137,17 & & 3,43 \\
142,16 & & 0,09 \\
149,64 & 142,04 & 5,35 \\
148,77 & & 4,74 \\
141,57 & & 0,33 \\
132,90 & & 6,44 \\
\hline
\end{tabular}

Qmáx: carga máxima, $\overline{Q_{\text {máx }}}$ : valor medio de Qmáx.

El valor mínimo $P_{\text {mín }}$ reducido en $90 \%$ de los 6 ensayos realizados por conector, es el valor de $P_{R K}$. Así, se realizó un análisis estadístico para cada uno de los conectores ensayados, donde se incluyó la dispersión de las cargas con respecto a su media y su mediana. En la Tabla 3 se muestran los valores de carga máxima por conector, los valores estadísticos y la resistencia característica obtenida de los 6 ensayos para el conector a $45^{\circ}$. Se realizó un análisis similar para el conector a $90^{\circ}$, obteniéndose una resistencia característica. En la Figuras 8 se presentan los valores de carga máxima experimental, con respecto a su media y mediana, para cada tipo de conector.

Tabla 3. Valor de $\mathrm{P}_{\mathrm{RK}}$ para conectores de corte tipo ángulo con posición a $45^{\circ}$.

\begin{tabular}{|c|c|c|c|c|}
\hline $\mathbf{N}^{\mathbf{o}}$ & $\begin{array}{c}\mathbf{Q} \\
{[\mathbf{k N}]}\end{array}$ & $\begin{array}{l}\text { Error } \\
(\%)\end{array}$ & $\begin{array}{c}\text { Desviación absoluta }^{1} \\
{[\mathrm{kN}]}\end{array}$ & $\begin{array}{c}\text { Desviación absoluta }^{2} \\
{[\mathrm{kN}]}\end{array}$ \\
\hline $45-1$ & 137,18 & 4,20 & 6,01 & 2,52 \\
\hline $45-2$ & 140,54 & 1,90 & 2,65 & 0,84 \\
\hline $45-3$ & 138,86 & 3,00 & 4,34 & 0,84 \\
\hline $45-4$ & 154,41 & 7,80 & 11,21 & 14,71 \\
\hline $45-5$ & 136,02 & 5,00 & 7,18 & 3,69 \\
\hline $45-6$ & 152,17 & 6,30 & 8,97 & 12,47 \\
\hline $\begin{array}{c}\mathbf{P}_{\text {mínimo }} \\
{[\mathrm{kN}]}\end{array}$ & $\begin{array}{c}\text { Media } \\
{[\mathrm{kN}]}\end{array}$ & $\begin{array}{c}\text { Mediana } \\
{[\mathrm{kN}]}\end{array}$ & $\begin{array}{l}\text { Desviación estándar } \\
{[\mathrm{kN}]}\end{array}$ & $\begin{array}{c}\mathbf{P}_{\text {RK }} \\
{[\mathrm{kN}]}\end{array}$ \\
\hline 136,02 & 143,20 & 139,70 & 8,00 & 122,41 \\
\hline
\end{tabular}

1 usa la media, ${ }^{2}$ usa la mediana, Q: carga máxima experimental, Pmínimo: mínimo valor de todas las capacidades máximas a corte obtenidas de las probetas ensayadas, $\mathrm{P}_{\mathrm{RK}}$ : resistencia característica a corte.

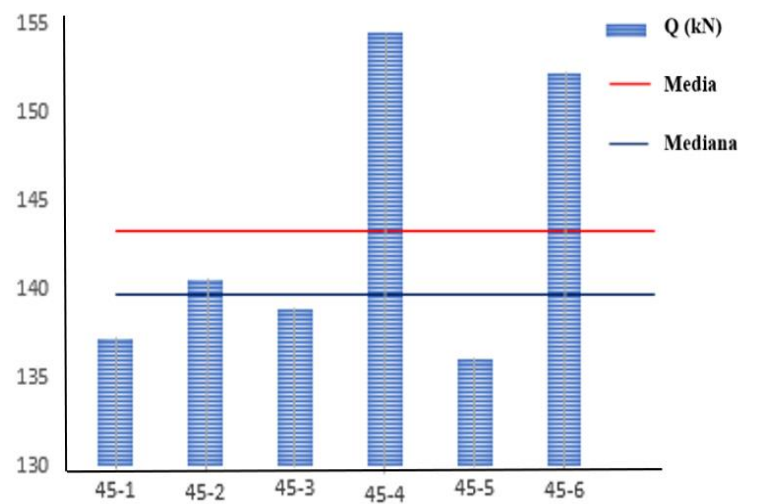

a) Conector a $45^{\circ}$

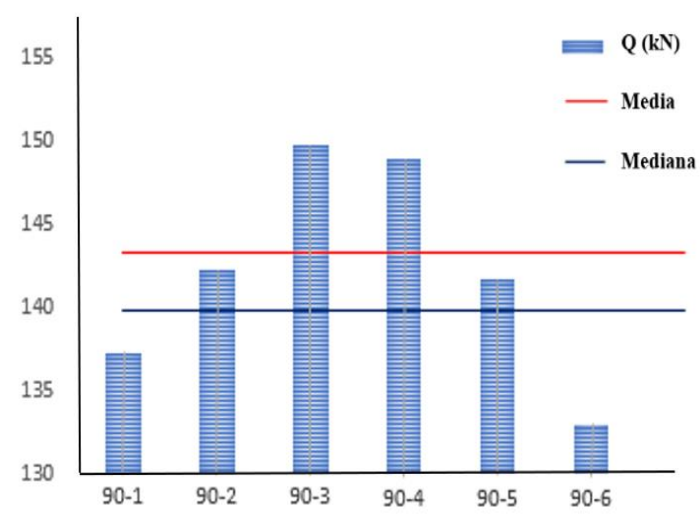

b) Conector a $90^{\circ}$

Figura 8. Valores de carga máxima experimental, con respecto a su media y mediana, para conectores tipo ángulo en posiciones a 45 y $90^{\circ}$. Q: carga máxima experimental.

Del análisis estadístico se obtuvo que el conector a $45^{\circ}$ presentó valores de carga máxima cercanos a su media y mediana; caso contrario al análisis del conector a $90^{\circ}$ (Tabla 4), en el cual el valor de la media y mediana tuvieron valores semejantes, presentando más probetas con una desviación absoluta distante. Además, presentó una resistencia característica de $122,41 \mathrm{kN}$, que resulta mayor a la obtenida del conector a $90^{\circ}$, con un valor de 119,61 $\mathrm{kN}$. 
Tabla 4. Valor de $\mathrm{P}_{\mathrm{RK}}$ para conectores de corte tipo ángulo con posición a $90^{\circ}$.

\begin{tabular}{ccccc}
\hline $\mathbf{N}^{\mathbf{0}}$ & $\begin{array}{c}\mathbf{Q} \\
{[\mathbf{k N}]}\end{array}$ & $\begin{array}{c}\text { Error } \\
(\boldsymbol{\%})\end{array}$ & $\begin{array}{c}\text { Desviación absoluta } \\
{[\mathbf{k N}]}\end{array}$ & $\begin{array}{c}\text { Desviación absoluta }^{\mathbf{2}} \\
{[\mathbf{k N}]}\end{array}$ \\
\hline $90-1$ & 137,20 & 4,2 & 5,99 & 2,50 \\
$90-2$ & 142,16 & 0,7 & 1,03 & 2,46 \\
$90-3$ & 149,64 & 4,5 & 6,44 & 9,94 \\
$90-4$ & 148,77 & 3,9 & 5,57 & 9,07 \\
$90-5$ & 141,57 & 1,1 & 1,63 & 1,87 \\
$90-6$ & 132,90 & 7,2 & 10,30 & 6,81 \\
$\mathbf{P}_{\text {mínimo }}$ & Media & Mediana & Desviación estándar & $\mathbf{P}_{\mathbf{R K}}$ \\
{$[\mathrm{kN}]$} & {$[\mathrm{kN}]$} & {$[\mathrm{kN}]$} & {$[\mathrm{kN}]$} & {$[\mathrm{kN}]$} \\
\hline 132,90 & 142,04 & 141,87 & 6,49 & 119,61 \\
\hline 13usa la media, ${ }^{2}$ usa la mediana, Q: carga máxima experimental, Pmínimo: mínimo valor de todas las capacidades \\
máximas a corte obtenidas de las probetas ensayadas, P $_{\mathrm{RK}}:$ resistencia característica a corte.
\end{tabular}

\section{Propiedades mecánicas}

Debido a que el ensayo push-out tiene una parte cíclica, la rigidez inicial de la curva carga-deslizamiento se vió afectada para el análisis de los conectores. Para determinar la rigidez inicial se utilizó el Eurocódigo 4 (UNE EN 1994 1-1, 2013), el cual considera el 40 \% de la capacidad máxima a corte de los conectores (Ecuación 1):

$$
\mathrm{K}_{\mathrm{o}}=\frac{40 \% \cdot Q_{\operatorname{máx}}}{\delta_{\mathrm{i}}}
$$

Donde: Qmáx es la capacidad máxima a corte de los conectores y $\delta$ i el deslizamiento registrado cuando el valor es de un tercio de la capacidad máxima.

El Eurocódigo 4 (UNE EN 1994 1-1, 2013) estipula el análisis de la resistencia característica para el 90\% de la capacidad máxima a corte, utilizando la ecuación de una rigidez secante, como lo indica la Ecuación 2:

$$
K=\frac{P_{R K}}{\delta_{i}}
$$

Donde: PRK es la capacidad característica a corte de los conectores y $\delta$ i el deslizamiento registrado cuando el valor es de la capacidad característica.

Por otra parte, Hällmark et al., (2019) determinaron la rigidez característica de acuerdo con los valores especificados en el Eurocódigo 4 (UNE EN 1994 1-1, 2013), con la reducción de la capacidad característica en un 75 $\%$ (Ecuación 3):

$$
K=\frac{0,75 \cdot P_{R K}}{\delta_{i}}
$$

Donde: PRK es la capacidad característica a corte de los conectores y $\delta$ i el deslizamiento registrado cuando el valor es de la capacidad característica.

Los valores de los coeficientes de la ductilidad de los conectores se pueden obtener de la curva carga vs. deslizamiento (Figura 9). Shariati et al., (2019) determinaron el factor de ductilidad o el coeficiente de la ductilidad $(\mu)$ con la división entre el deslizamiento característico $(\delta \mathrm{u})$ y el deslizamiento a fluencia $(\delta \mathrm{y})$, para cada conector ensayado (Ecuación 4):

$$
\mu=\frac{\delta_{u}}{\delta_{y}}
$$




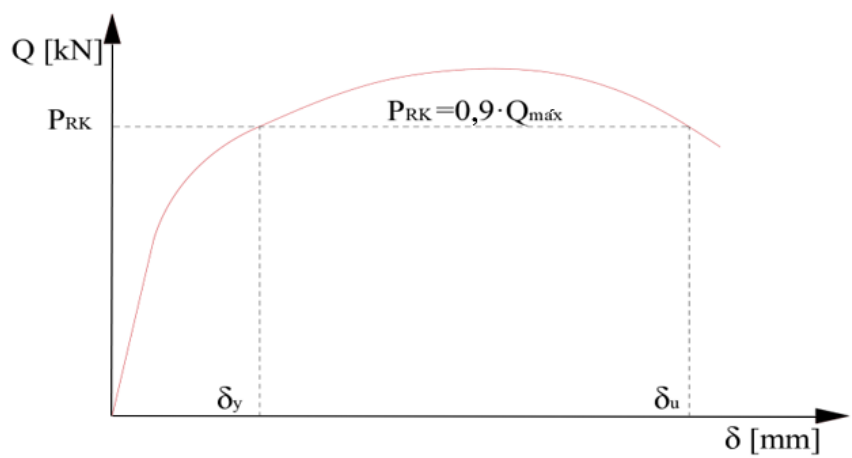

Figura 9. Curva carga-deslizamiento, conector a 90. PRK: resistencia característica a corte, Qmáx: carga máxima.

La tenacidad de los conectores de corte se determinó como el área acumulada bajo la curva cargadeslizamiento de los conectores de corte hasta antes de llegar a la falla por rotura, se emplea el método del trapecio para determinar el área bajo la curva, Tablas 5 y 6.

Tabla 5. Factor de ductilidad, tenacidad y rigidez para conectores de corte tipo ángulo con posición a $45^{\circ}$.

\begin{tabular}{cccccccc}
\hline $\mathbf{N}^{\mathbf{o}}$ & $\begin{array}{c}\mathbf{\delta u} \\
{[\mathbf{m m}]}\end{array}$ & $\begin{array}{c}\mathbf{\Delta y} \\
{[\mathbf{m m}]}\end{array}$ & $\begin{array}{c}\mathbf{U} \\
\text { factor }\end{array}$ & $\begin{array}{c}\text { Tenacidad } \\
{[\mathbf{J}]}\end{array}$ & $\begin{array}{c}\mathbf{k}, \mathbf{9 0} \% \cdot \mathbf{Q}_{\text {máx }} \\
{[\mathbf{k N} / \mathbf{m m}]}\end{array}$ & $\begin{array}{c}\mathbf{k , ~ 7 5 ~ \%} \cdot \mathbf{P}_{\mathbf{R K}} \\
{[\mathbf{k N} / \mathbf{m m}]}\end{array}$ & $\begin{array}{c}\mathbf{k}, \mathbf{4 0} \% \cdot \mathbf{Q}_{\text {máx }} \\
{[\mathbf{k N} / \mathbf{m m}]}\end{array}$ \\
\hline $45-1$ & 13,27 & 3,86 & 3,44 & 1879,74 & 32,00 & 67,18 & 132,75 \\
$45-2$ & 14,04 & 5,05 & 2,78 & 2038,42 & 25,06 & 76,18 & 188,06 \\
$45-3$ & 13,37 & 4,86 & 2,75 & 1766,90 & 25,70 & 63,89 & 140,72 \\
$45-4$ & 12,89 & 5,01 & 2,57 & 1794,82 & 27,75 & 68,29 & 134,37 \\
$45-5$ & 12,28 & 3,98 & 3,08 & 1750,47 & 30,73 & 96,90 & 198,93 \\
$45-6$ & 12,81 & 5,12 & 2,50 & 1796,00 & 26,72 & 68,69 & 166,10 \\
$\begin{array}{c}\text { Media } \\
\text { Desviación } \\
\text { estándar }\end{array}$ & 13,11 & 4,65 & 2,85 & 1837,73 & 27,99 & 73,52 & 160,16 \\
\hline
\end{tabular}

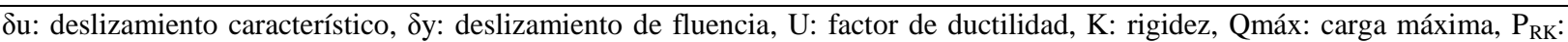
resistencia característica a corte.

En la Tabla 5 se presentan los valores de ductilidad, tenacidad y rigidez de los conectores a $45^{\circ}$. Del análisis de la resistencia de diseño, la probeta 45-5 exhibió el mínimo valor de carga máxima de los 6 ensayos. Se observó que la rigidez en el $90 \%$ de $Q_{\text {máx }}$ fue menor que la rigidez en el $40 \%$ de $Q_{\text {máx }}$, debido al incremento de deslizamiento.

Tabla 6. Factor de ductilidad, tenacidad y rigidez para conectores de corte tipo ángulo con posición a $90^{\circ}$.

\begin{tabular}{cccccccc}
\hline $\mathbf{N}^{\mathbf{0}}$ & $\begin{array}{c}\boldsymbol{\delta} \mathbf{u} \\
{[\mathbf{m m}]}\end{array}$ & $\begin{array}{c}\boldsymbol{\delta} \mathbf{m m} \\
{[\mathbf{m m}]}\end{array}$ & $\begin{array}{c}\mathbf{U} \\
\text { factor }\end{array}$ & $\begin{array}{c}\text { Tenacidad } \\
{[\mathbf{J}]}\end{array}$ & $\begin{array}{c}\mathbf{k}, \mathbf{9 0} \% \cdot \mathbf{Q}_{\mathbf{m a ́ x}} \\
{[\mathbf{k N} / \mathbf{m m}]}\end{array}$ & $\begin{array}{c}\mathbf{k}, \mathbf{7 5} \% \cdot \mathbf{P}_{\mathbf{R K}} \\
{[\mathbf{k N} / \mathbf{m m}]}\end{array}$ & $\begin{array}{c}\mathbf{k}, \mathbf{4 0} \% \cdot \mathbf{Q}_{\text {máx }} \\
{[\mathbf{k N} / \mathbf{m m}]}\end{array}$ \\
\hline $90-1$ & 25,62 & 8,82 & 2,90 & 3207,20 & 16,50 & 53,87 & 616,89 \\
$90-2$ & 13,10 & 3,65 & 3,59 & 1699,50 & 35,04 & 102,65 & 643,49 \\
$90-3$ & 17,03 & 5,74 & 2,97 & 2006,19 & 23,47 & 69,20 & 341,38 \\
$90-4$ & 17,08 & 5,55 & 3,07 & 2253,88 & 24,10 & 71,58 & 339,86 \\
$90-5$ & 14,00 & 5,09 & 2,75 & 1694,16 & 25,02 & 66,73 & 283,10 \\
$90-6$ & 11,86 & 3,31 & 3,58 & 1568,33 & 36,09 & 119,47 & 620,97 \\
$\begin{array}{c}\text { Media } \\
\text { Desviación }\end{array}$ & 16,45 & 5,36 & 3,14 & 2071,54 & 26,70 & 80,59 & 474,28 \\
estándar & 4,96 & 1,97 & 0,36 & 610,13 & 7,51 & 24,96 & 168,98 \\
\hline
\end{tabular}

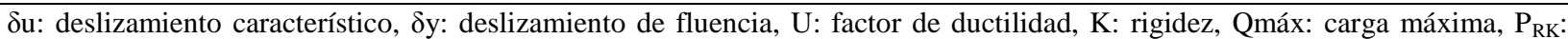
resistencia característica a corte.

De acuerdo con los deslizamientos vistos entre ambos conectores y respecto al factor de ductilidad media, presentados en las Tablas 5 y 6, se determinó que el valor de la ductilidad del conector a $90^{\circ}$ fue mayor en un 10,17 
$\%$, con relación al conector de $45^{\circ}$, no obstante, esto no indica que esta último tiene un mejor comportamiento. Esto se debe a que presentó mayores desviaciones estándares de los desplazamientos en el conector a $90^{\circ}$, lo que no mostró el conector de $45^{\circ}$, ya que los valores no difieren mucho entre sí, para los 3 parámetros analizados.

De las tenacidades mostradas en las Tablas 5 y 6 , se deduce que el conector a $45^{\circ}$ tuvo una energía absorbida media de $1837,73 \mathrm{~J}$, mientras que el conector de $90^{\circ}$ presentó un valor de $2071,54 \mathrm{~J}$. Aunque los valores del conector a $90^{\circ}$ son mayores que el del $45^{\circ}$, se puede llegar a realizar el mismo análisis con la desviación estándar de los valores obtenidos. Además, observando las curvas de carga vs. deslizamiento, y de acuerdo con el Eurocódigo 4 (UNE EN 1994 1-1, 2013), se establece que la ductilidad del conector a 45 es dúctil.

\section{Análisis de la capacidad a corte}

Se hace necesario encontrar un modelo numérico que se asemeje a los resultados obtenidos de los ensayos push-out. Para este análisis se utilizó la Ecuación 5, planteada por el ANSI/AISC 360-16 (2016). Así mismo, se presenta esta ecuación aplicando el estado límite de resistencia estipulado por el AASHTO-LRFD (2014) y el factor de reducción, cuyo valor fue de 0,85 ( $\left.\phi_{\mathrm{sc}}\right)$ (Ecuación 6). A saber:

$$
\begin{aligned}
& Q_{n}=0,5 \cdot A_{s a} \cdot \sqrt{E_{c} \cdot f_{c}^{\prime}} \\
& Q_{A A S H T O-L R F D}=\phi_{s c} \cdot Q_{n}
\end{aligned}
$$

El Eurocódigo 4 (UNE EN 1994 1-1, 2013) presenta la Ecuación 7 donde se emplean los elementos del conector de corte tipo stud, como el diámetro del perno, d $(\mathrm{mm})$, y la altura del conector, $\mathrm{h}_{\mathrm{sc}}(\mathrm{mm})$, además, la resistencia a compresión del hormigón, $\mathrm{f}_{\mathrm{c}}{ }_{\mathrm{c}}\left(\mathrm{N} / \mathrm{mm}^{2}\right)$, el módulo de elasticidad, $\mathrm{E}_{\mathrm{c}}\left(\mathrm{N} / \mathrm{mm}^{2}\right)$ y el coeficiente alfa $(\alpha)$ que debe ser elegido como el menor valor de las Ecuaciones 8 y 9 :

$$
\begin{gathered}
Q_{E N-4}=\frac{0,29 \cdot \alpha \cdot d^{2} \cdot \sqrt{f^{\prime} c^{\cdot} E_{c}}}{1,5} \\
\alpha=0,2 \cdot\left(\frac{h_{s c}}{d}+1\right) \text { cuando } 3 \leq h_{s c} / d \leq 4 \\
\alpha=1 \text { cuando } h_{s c} / d>4
\end{gathered}
$$

El código de estructuras de acero de la República de China (GB-50017, 2017), incluye la Ecuación 10 para el diseño de conectores tipo ángulo, donde el espesor del alma del conector es $\mathrm{t}_{\mathrm{w}}(\mathrm{mm})$, el espesor del patín $\mathrm{t}_{\mathrm{f}}(\mathrm{mm})$, el ancho del conector $\mathrm{L}_{\mathrm{c}}(\mathrm{mm})$ y las propiedades del hormigón $\mathrm{E}_{\mathrm{c}} \mathrm{y} \mathrm{f}_{\mathrm{c}_{\mathrm{c}}} \quad\left(\mathrm{N} / \mathrm{mm}^{2}\right)(\mathrm{GB} 50017,2017)$ :

$$
Q_{G B-50017}=0,26 \cdot\left(t_{f}+0,5 \cdot t_{w}\right) \cdot L_{c} \cdot \sqrt{E_{c} \cdot f^{\prime}}
$$

Los autores Bamaga et al. (2019) emplearon la Ecuación 11 diferente a los códigos antes mencionados, que utiliza la geometría del conector tipo ángulo, como su altura $\mathrm{h}_{\mathrm{sc}}(\mathrm{mm})$ y el espesor $\mathrm{t}_{\mathrm{sc}}(\mathrm{mm})$, además de la resistencia del hormigón $\mathrm{f}_{\mathrm{c}}^{\prime}\left(\mathrm{N} / \mathrm{mm}^{2}\right)$ :

$$
Q=4,5 \cdot h_{s c} \cdot t_{s c} \cdot f^{\prime}{ }_{c}
$$

Titoum et al. (2016) obtuvieron excelentes resultados aplicando la Ecuación 12, dictada por la norma canadiense (CSA-S16-0, 2001) para determinar la capacidad máxima a corte de los conectores tipo I; una ecuación similar presenta el ANSI/AISC 360-16 (2016) para conectores tipo canal. Se empleó la Ecuación 8, usada por Titoum et al. (2016) para una viga I con momento de inercia similar a la del conector en ángulo. La viga que se asemeja tenía un espesor de $4 \mathrm{~mm}$ para el alma ( $\mathrm{t}_{\mathrm{f}}$ ) y de $4 \mathrm{~mm}$ para el ala ( $\mathrm{t}_{\mathrm{w}}$ ) (Titoum et al., 2016):

$$
Q=36,5 \cdot\left(t_{f}+0,5 \cdot t_{w}\right) \cdot b \cdot \sqrt{f_{c}^{\prime}}
$$

Las capacidades a corte determinados con las diferentes investigaciones se detallan en la Tabla 7. 
Tabla 7. Capacidad a corte para conectores de corte tipo ángulo con posiciones de 45 y $90^{\circ}$.

\begin{tabular}{|c|c|c|c|}
\hline Norma & $\mathbf{Q}[\mathrm{kN}]$ & Qe [kN] & Error (\%) \\
\hline \multicolumn{4}{|c|}{ Conector a $45^{\circ}$} \\
\hline ANSI/AISC 360-16 (2016) & 115,36 & 143,2 & 24,13 \\
\hline AASHTO-LRFD (2014) & 98,06 & 143,2 & 46,03 \\
\hline UNE-EN-1994 (2013) & 56,78 & 143,2 & 152,20 \\
\hline GB-50017 (2017) & 66,11 & 143,2 & 116,61 \\
\hline Bamaga et al. (2019) & 38,19 & 143,2 & 274,97 \\
\hline Titoum et al. (2016) & 63,8 & 143,2 & 124,45 \\
\hline \multicolumn{4}{|c|}{ Conector a $90^{\circ}$} \\
\hline ANSI/AISC 360-16 (2016) & 115,36 & 146,13 & 26,67 \\
\hline AASHTO-LRFD (2014) & 98,06 & 146,13 & 49,02 \\
\hline UNE-EN-1994 (2013) & 56,78 & 146,13 & 157,36 \\
\hline GB-50017 (2017) & 46,74 & 146,13 & 212,64 \\
\hline Bamaga et al. (2019) & 38,19 & 146,13 & 282,64 \\
\hline Titoum et al. (2016) & 45,11 & 146,13 & 223,94 \\
\hline
\end{tabular}

\section{Conclusiones}

El comportamiento de los conectores de corte tipo ángulo para 45 y $90^{\circ}$ es considerado como dúctil, de acuerdo con el deslizamiento característico que presentaron las 12 probetas para el ensayo push out. Los resultados de las ecuaciones propuestas en investigaciones previas y códigos internacionales, al ser adaptadas y empleadas únicamente con el fin de comparar los resultados experimentales de este estudio, muestran que subestiman el valor de resistencia del conector, por lo que se recomienda mayor investigación previa a establecer una ecuación que estime la resistencia de este tipo de conector.

De los 12 ensayos realizados de los conectores de corte con dimensión de 40 x $4 \mathrm{~mm}$ y con las consideraciones antes expuestas, el comportamiento de los conectores fue determinado exitosamente mediante el ensayo experimental; donde se determinó que la posición a $45^{\circ}$ cumple con un comportamiento más homogéneo en todos los parámetros analizados que fueron obtenidos de las curvas. Su óptimo rendimiento hace que este conector sea el más adecuado para su implementación en futuras investigaciones, de manera apropiada y técnica en el ámbito de la construcción.

\section{Referencias Bibliográficas}

AASHTO LRFD. (2014). Bridge design specifications. Washington DC: American Association of State Highway and Transportation Officials (ASSHTO).

ANSI/AISC 360-16. (2016). Specification for structural steel buildings. Illinois: American Institute of Steel Construction (AISC).

Bamaga, S. O., Tahir, M. M., Tan, C. S., Shek, P. N., Aghlara R. (2019). Push-out tests on three innovative shear connectors for composite cold-formed steel concrete beams. Construction and Building Materials, 223, 288-298.

Chen, J., Jiang, A. Y.- Jin, W. L. (2016). Behaviour of corroded stud shear connectors under fatigue loading. $5^{\text {th }}$ International conference on durability of concrete structures (ICDCS 2016). No. 2004, 127-133.

Crisafulli, F. (2018). Diseño sismoresistente de construcciones de acero. Mendoza: Asociación Latinoamericana de Acero.

CSA-S16-0. (2001). Limit states sesign of steel structures. Ontario: Canadian Standards Association (CSA).

Deng, W., Xiong, Y., Liu, D., Zhang, J. (2019). Static and fatigue behavior of shear connectors for a steel-concrete composite girder. Journal of Constructional Steel Research, 159, 134-146.

GB 50017. (2017). Code for design of steel structures. Beijing: Ministry of Constructions of the People's Republic 
of China.

Hällmark, R., Collin, P., Hicks, S. J. (2019). Post-installed shear connectors: Push-out tests of coiled spring pins vs. headed studs. Journal of Constructional Steel Research, 161, 1-16.

Horita, Y., Tagawa, Y., Hayato, A. (2012). Push-out test of headed stud in composite girder using steel deck - An effect of stud length of projecting part from steel deck on shear strength. Proceedings of the $15^{\text {th }}$ world conference on earthquake engineering (15 WCEE Lisboa 2012), 18762-18771.

McCormac, J., Csernak, S. (2013). Diseño de estructuras de acero. $5^{\text {ta }}$ ed. Mexico: Alfaomega Grupo Editor S.A. de C.V.

Molina, M., Hurtado, X. (2011). Formulación para el diseño de conectores de cortante tipo tornillo en secciones compuestas, 31(2), 52-64.

Pavlović, M., Marković, Z., Veljković, M., Bucrossed D Dignevac, D. (2013). Bolted shear connectors vs. headed studs behaviour in push-out tests. Journal of Constructional Steel Research, 88, 134-149.

Shariati, A., Shariati, M., Ramli Sulong, N. H., Arabnejad Khanouki, M. M. y Mahoutian, M. (2014). Experimental assessment of angle shear connectors under monotonic and fully reversed cyclic loading in high strength concret. Construction and Building Materials, 52, 276-283.

Titoum, M., Mazoz, A., Benanane, A., Ouinas, D. (2016). Experimental study and finite element modelling of pushout tests on a new shear connector of I-shape. Advanced Steel Construction, 12, 4, 487-506.

UNE EN 1990. (2019). Eurocode 0 - Basis of structural design. Madrid: European Comittee for Standardization (CEN).

UNE EN 1994 1-1. (2013). Eurocode 4 - Desing of composite steel and conncrete structures- Part 1-1: General rules and rules for buildings. Madrid: European Comittee for Standardization (CEN). 


\section{REVISTA TECNICA}

DE LA FACULTAD DE INGENIERIA

UNIVERSIDAD DEL ZULIA

Vol. 45. №1, Enero - Abril, 2022

Esta revista fue editada en formato digital y publicada en Diciembre 2021, por el Fondo Editorial Serbiluz, Universidad del Zulia. Maracaibo-Venezuela

www.luz.edu.ve

www.serbi.luz.edu.ve

www.produccioncientificaluz.org 